\title{
A NOTE ON IRREDUCIBLE HEEGAARD DIAGRAMS
}

\author{
ALBERTO CAVICCHIOLI AND FULVIA SPAGGIARI
}

Received 19 December 2005; Revised 26 April 2006; Accepted 7 May 2006

We construct a Heegaard diagram of genus three for the real projective 3-space, which has no waves and pairs of complementary handles. The first example was given by Im and Kim but our diagram has smaller complexity. Furthermore the proof presented here is quite different to that of the quoted authors, and permits also to obtain a simple alternative proof of their result. Examples of irreducible Heegaard diagrams of certain connected sums complete the paper.

Copyright (c) 2006 Hindawi Publishing Corporation. All rights reserved.

\section{Introduction}

The construction of an algorithm for recognizing the standard 3-sphere $\mathbb{S}^{3}$ is a very important problem in the topology of closed 3-manifolds. The first work in this direction was done by Whitehead [28] who discovered that certain Heegaard diagrams of $\mathbb{S}^{3}$ have a special geometric property (see [28, Conjecture A]). Later Volodin et al. [27] conjectured that every Heegaard diagram of $\mathbb{S}^{3}$ is reducible by wave moves (see Section 2 for the definition), except for the canonical one. Really, the conjecture is true for the case of genus two, as proved by Homma et al. [10]. But it is not true for genera greater than two. For genus 3 diagrams the conjecture was first disproved by Viro and Kobel'skiu [26]. Further counterexamples were given by Morikawa [14] for the case of genus three, and Ochiai $[17,18]$ for the case of genera three and four. In [15] Negami proved that every 3-bridge projection of a link can be transformed into a minimum crossing one by a finite sequence of wave moves if and only if the link is equivalent to one of the following: a trivial knot, a splittable link, or the Hopf link (see also [16]). It follows from [1] that any result on wave moves for 3-bridge projections of a link can be translated into one on wave moves for genus two Heegaard diagrams of a closed orientable 3-manifold (represented as the 2 -fold covering of $\mathbb{S}^{3}$ branched over the link). So we have the following theorem.

Theorem $1.1[10,15,16]$. Any Heegaard diagram of genus two for $\mathbb{S}^{3}, \mathbb{R} P^{3}$, or $\left(\mathbb{S}^{1} \times\right.$ $\left.\mathbb{S}^{2}\right) \# L(p, q)$, except for the canonical ones of genus two, is reducible, and can be transformed into one of the specific standard forms by a finite sequence of wave moves. 
This result cannot be extended to Heegaard diagrams of genus three for $\mathbb{S}^{3}$ and $\mathbb{R} P^{3}$ (see $[11,14,17,18])$. In the present paper we construct a Heegaard diagram of genus three for $\mathbb{R} P^{3}$, which has no waves and pairs of complementary handles (see Section 3 ). The complexity of our diagram is less than the complexity of the diagram given by Im and Kim in [11]. The proof that the 3-manifold determined by the diagram is $\mathbb{R} P^{3}$ is based on the fact that the diagram is invariant under an orientation preserving involution of the Heegaard surface. Thus the corresponding 3-manifold is a 2-sheeted branched covering of $\mathbb{S}^{3}$. This method was first used by Viro and Kobel'skii [26]. The same proof works also for the example of Im and Kim, and permits to obtain a simple alternative proof of their result (see Section 4). Examples of irreducible Heegaard diagrams of genus three for connected sums of $\mathbb{R} P^{3}$ and lens spaces are given in Section 5 .

\section{Wave moves and Heegaard diagrams}

Concepts and notations from the theory of Heegaard splittings and diagrams are standard and can be found, for example $[6,8,22]$. Following $[11,17]$, we recall briefly some definitions and preliminaries on reducible Heegaard diagrams and wave moves. Let $M$ be a closed connected orientable 3-manifold, $W_{1}$ and $W_{2}$ solid tori of genus $n$, and $h: \partial W_{2} \rightarrow \partial W_{1}$ a homeomorphism of the boundary surfaces. Then the triple $\left(W_{1}, W_{2} ; h\right)$ is said to be a Heegaard splitting of genus $n$ for $M$ if $M=W_{1} \cup_{h} W_{2}$. A properly embedded disk $D$ in a solid torus $W$ of genus $n$ is called a meridian disk of $W$ if $\operatorname{cl}(W \backslash N(D, W))$ is a solid torus of genus $n-1$. Here $N(D, W)$ denotes a regular neighborhood of $D$ in $W$. A collection of mutually disjoint $n$ meridian disks $D_{1}, \ldots, D_{n}$ in $W$ is called a complete system of meridian disks of $W$ if $\mathrm{cl}\left(W \backslash \cup_{i=1}^{n} N\left(D_{i}, W\right)\right)$ is a 3-ball. Let $\left\{D_{i 1}, \ldots, D_{i n}\right\}$ be a complete system of meridian disks of $W_{i}, i=1,2$, and let $u_{j}=\partial D_{1 j}$ and $v_{j}^{\prime}=\partial D_{2 j}$ for $j=1, \ldots, n$. Then the manifold $M=W_{1} \cup_{h} W_{2}$ is determined, up to homeomorphism, by the collection of circles $v_{1}, \ldots, v_{n}$ on $\partial W_{1}$ with $v_{j}=h\left(v_{j}^{\prime}\right)$ for $j=1, \ldots, n$. The triple $(F ; u, v)$ is called a Heegaard diagram of genus $n$ for $M$, where $F=\partial W_{1}$ is the splitting surface, $u=u_{1} \cup \cdots \cup u_{n}$, and $v=v_{1} \cup \cdots \cup v_{n}$. Of course, there are many different Heegaard diagrams associated with a given Heegaard splitting of genus $\geq 2$; a nice discussion on the structure of Heegaard diagrams of genus two can be found in [6, Chapter 5]. A fundamental result of the theory states that two Heegaard diagrams represent homeomorphic 3-manifolds if and only if one of them can be obtained from the other by a finite sequence of elementary moves, called the Singer moves [23]. So Heegaard diagrams, up to Singer moves, one-to-one correspond to closed connected orientable 3-manifolds, up to homeomorphism. Relations between Heegaard diagrams and branched coverings can be found in [1]. Here it was proved that there is a bijective correspondence between the equivalence classes of 3-bridge links with decomposing spheres and those of Heegaard splittings of genus two. Now we recall the concept of wave for a Heegaard diagram. Let $H=(F ; u, v)$ be a Heegaard diagram of a closed connected orientable 3-manifold $M$. By definition, the complexity $c(H)$ of $H$ is the cardinality of the set $u \cap v$. Let us consider an $\operatorname{arc} w$ on $F$ such that for a meridian or a longitude of $H, u_{1}$ say, $w \cap(u \cup v)=w \cap u_{1}=\partial w$ and both ends of $w$ attach to the same side of $u_{1}$. Then one of the two circles, $u_{1}^{\prime}$ say, in $u_{1} \cup w$, different from $u_{1}$, bounds a meridian disk of $H$, and $H^{\prime}=\left(F ; u^{\prime}, v\right)$ is a new 


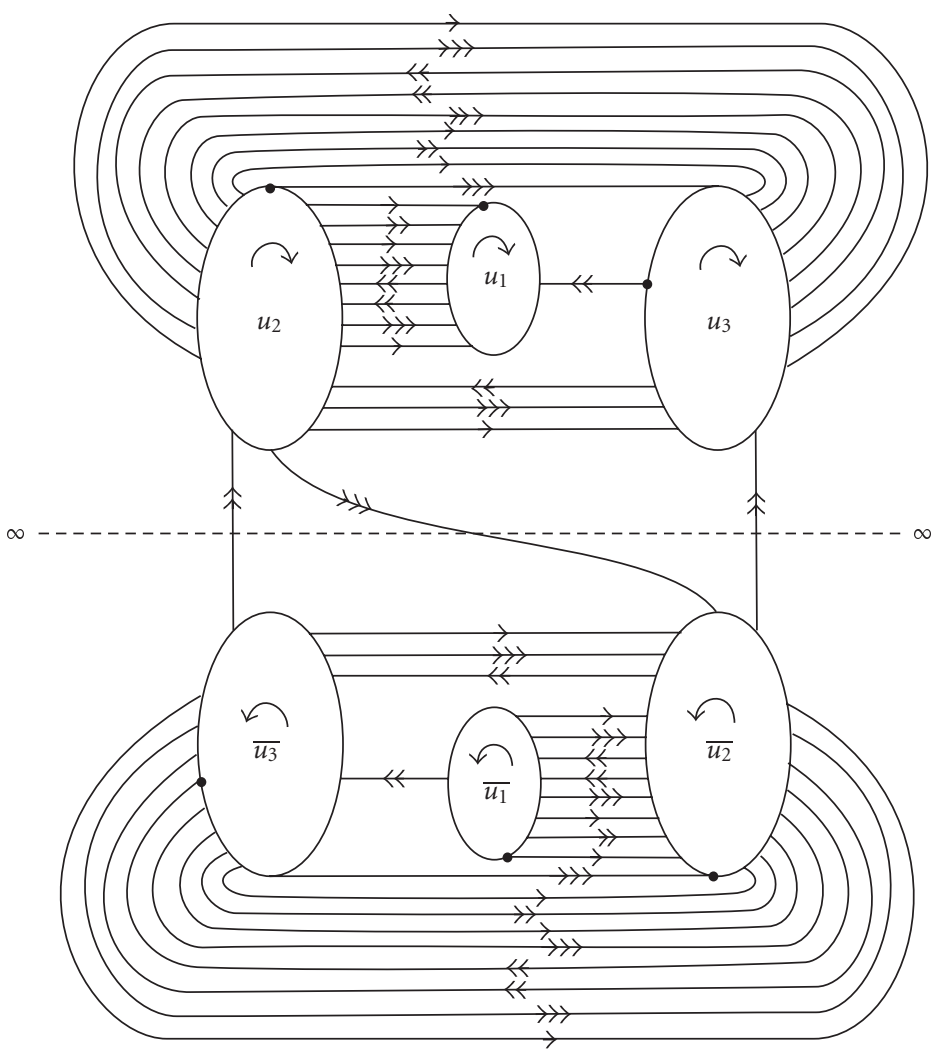

Figure 3.1. An irreducible Heegaard diagram of genus three for $M^{3}$.

Heegaard diagram of $M$, where $u^{\prime}=u_{1}^{\prime} \cup u_{2} \cup \cdots \cup u_{n}$. We say that the arc $w$ is a wave for $H$, and that the replacement of $u_{1}$ with $u_{1}^{\prime}$ is a wave move of $H$ if $c\left(H^{\prime}\right)<c(H)$. A Heegaard diagram is said to be reducible if it contains at least one wave. We say that a Heegaard diagram is irreducible if it has no waves and pairs of complementary handles.

\section{An irreducible Heegaard diagram for $\mathbb{R} P^{3}$}

In this section we construct a Heegaard diagram of genus three for the real projective 3space, which has no waves and pairs of complementary handles. Our example has complexity 45 while that constructed in [11, Figure 1], has complexity 49 (see also Figure 4.1). Let us consider the Heegaard diagram of genus three, depicted in Figure 3.1. The Heegaard surface $F$ is obtained from the picture in Figure 3.1 by identifying the oriented meridians $u_{i}$ with $\bar{u}_{i}, i=1,2,3$, so that the marked vertices and orientations coincide. It is easily checked that this diagram has no waves and pairs of complementary handles. 


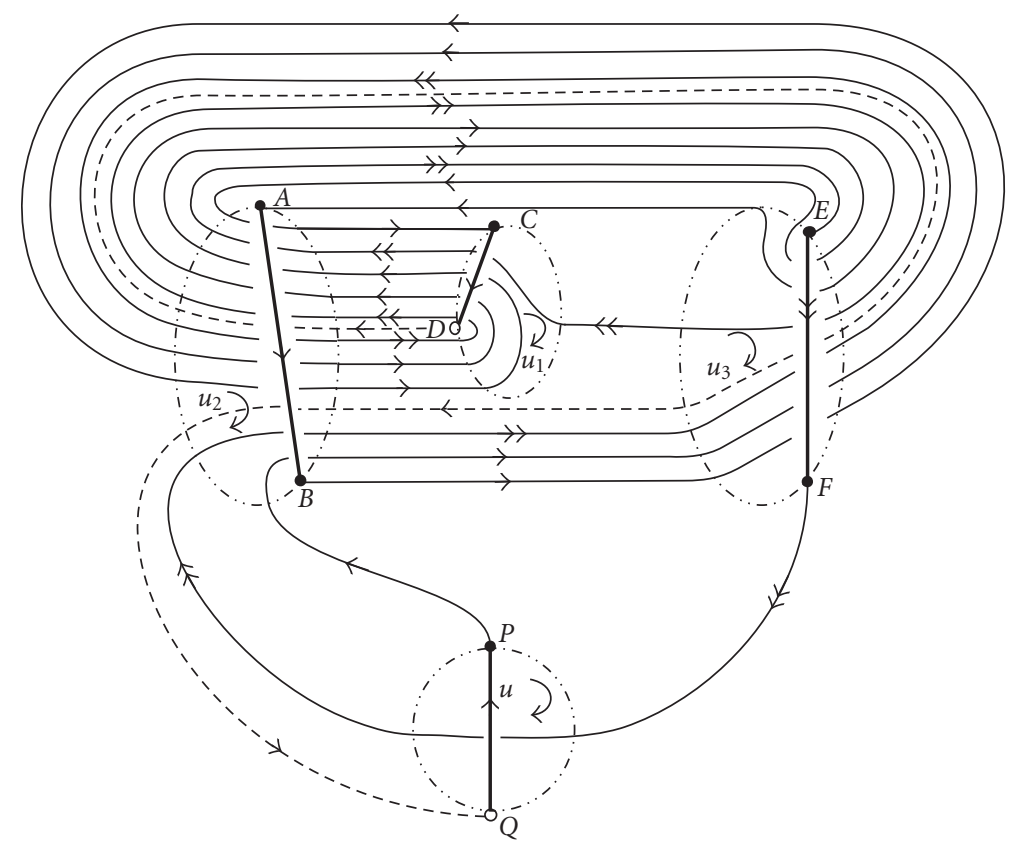

Figure 3.2. Determining the branch set of a representation of $M^{3}$ as 2 -fold branched covering.

Theorem 3.1. Let $M^{3}$ be the closed connected orientable 3-manifold represented by the irreducible Heegaard diagram of genus three, depicted in Figure 3.1. Then $M^{3}$ is homeomorphic to the real projective 3-space $\mathbb{R} P^{3}$.

Proof. We describe $M^{3}$ as 2-fold branched covering of the 3-sphere by using standard constructions explained in $[1,24,26]$. We consider the 3-disk whose boundary is the 2-sphere $\mathbb{S}^{2}=\mathbb{R}^{2} \cup\{\infty\}$ containing the planar representation of our Heegaard diagram. Then we cut the 3-disk through the interior 2-disk with boundary represented by the dotted horizontal axis in Figure 3.1 (in fact, its compactification at infinity). So the considered 3-disk is divided into two smaller 3-disks. We represent only one of them in the diagram pictured in Figure 3.2. Then we construct three symmetric axes (the marked lines in Figure 3.2), and connect the symmetric points with respect to these lines. We can regard this picture as the union of a circle $\alpha$ and a simple arc $\beta$ in the 3 -sphere. The circle $\alpha$ is formed by two oriented parts, that is, the oriented upper path $E F$ and the lower path $F E$. The arc $\beta$ is formed by five oriented parts, that is, the oriented upper path $Q P$, the lower path $P A$, the upper path $A B$, the lower path $B C$, and the upper path $C D$. If we connect $D$ with $Q$ by a suitable lower path (i.e., the dotted oriented lower path $D Q$ in Figure 3.2), we get the 2-component link drawn in Figure 3.3(a). The sequence of Reidemeister moves in Figure 3.3 proves that this link is equivalent to the Hopf link. So the manifold $M^{3}$ is homeomorphic to the real projective 3-space $\mathbb{R} P^{3}$.

The Heegaard diagram in Figure 3.1 determines a balanced geometric presentation for $\pi_{1}\left(M^{3}\right) \cong \mathbb{Z}_{2}$ with three generators $X, Y$, and $Z$ corresponding to meridians $u_{1}, u_{2}$ and $u_{3}$, 


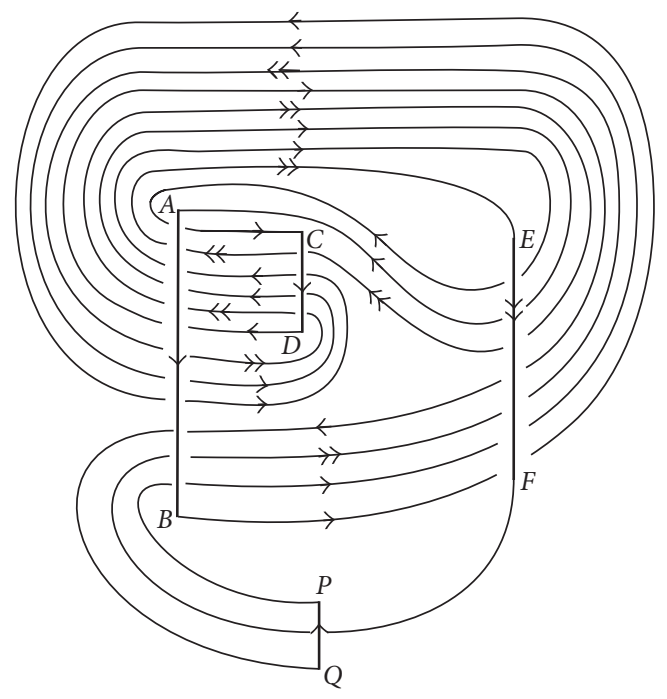

(a)

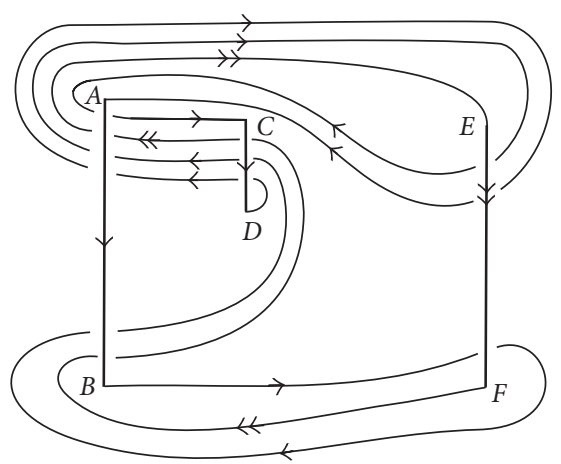

(b)

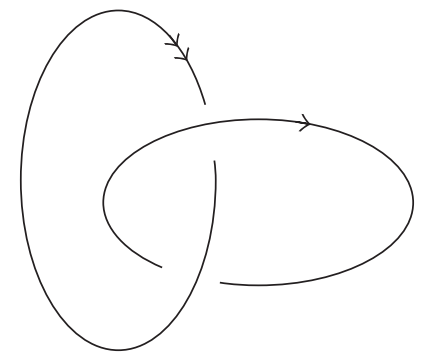

(c)

Figure 3.3. A sequence of Reidemeister moves yielding the Hopf link. 


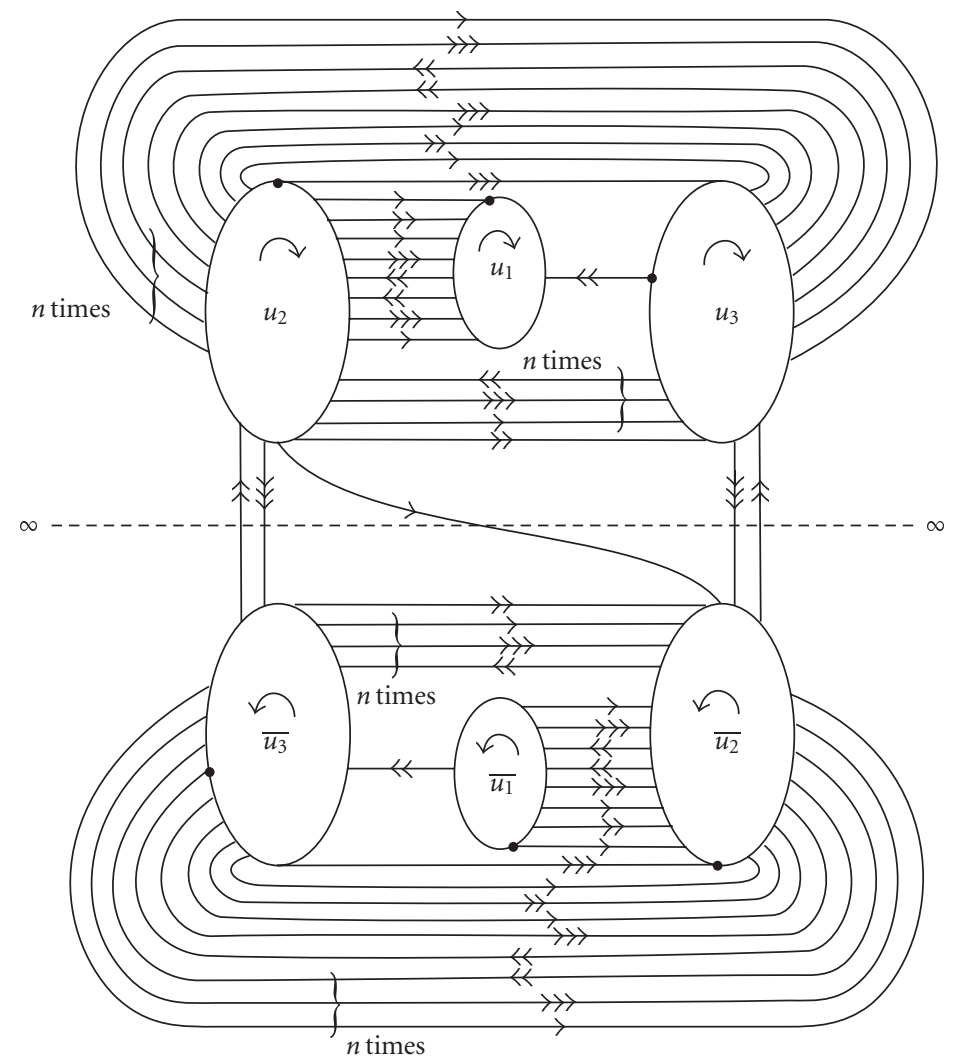

Figure 4.1. The irreducible diagram of $\operatorname{Im}$ and $\operatorname{Kim}(n=1)$.

respectively, and three relations

$$
\begin{gathered}
Y X^{-1}\left(Y Z^{-1} Y X^{-1} Y Z^{-1}\right)^{2}=1, \\
Y\left(Y Z^{-1} Y X^{-1} Y Z^{-1}\right)^{2}=1, \\
Y X^{-1} Z Y^{-1} X Y^{-1} Z Y^{-1} Z^{-1} Y^{-1} Z Y^{-1} X Y^{-1} Z X^{-1} Y Z^{-1}=1,
\end{gathered}
$$

which correspond to longitudes. This presentation also arises from a spine of the manifold (we refer to [21] for a classification of 3-thickenings of 2-polyhedra).

\section{The irreducible diagram of Im and Kim}

The irreducible Heegaard diagram of genus three, given by Im and Kim in [11], is illustrated in Figure 4.1. The next theorem was proved in [11] by using the combinatorial representation of closed manifolds via colored graphs (and crystallizations-a special class of them). See for example $[2-5,7,19,20]$ for information about this theory. Here we give 


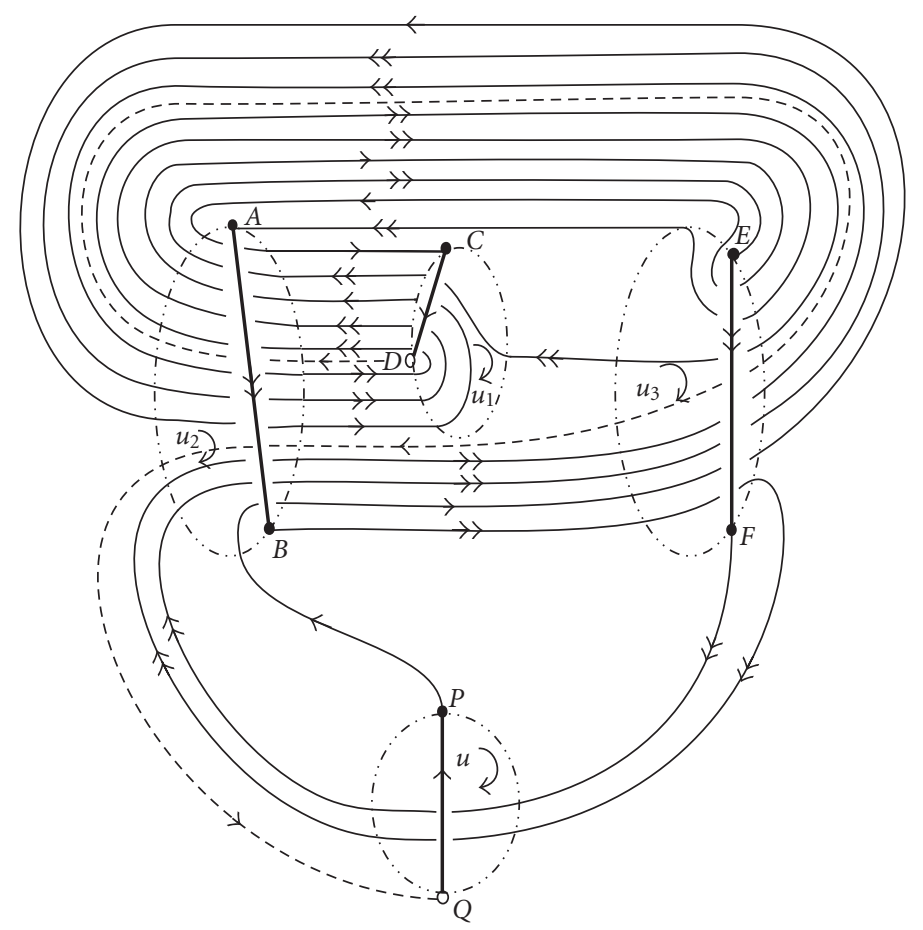

Figure 4.2. The branch set arising from the 2-symmetric diagram of Im and Kim.

a simple alternative proof of the theorem which is intrinsic to the theory of Heegaard diagrams.

THeOREM 4.1 [11]. Let $M^{3}$ be the closed connected orientable 3-manifold represented by the irreducible Heegaard diagram of genus three, depicted in Figure 4.1. Then $M^{3}$ is homeomorphic to the real projective 3-space $\mathbb{R} P^{3}$.

Proof. We will proceed as in the proof of Theorem 3.1. Determining the branch set of a representation of $M^{3}$ as a 2-fold covering of the 3-sphere yields Figure 4.2. We can regard this picture as the union of a circle $\alpha$ and a simple arc $\beta$ in the 3 -sphere. The circle $\alpha$ is formed by four oriented parts, that is, the oriented upper path $A B$, the lower path $B E$, the upper path $E F$, and the lower path $F A$. The $\operatorname{arc} \beta$ is formed by three oriented parts, that is, the oriented upper path $Q P$, the lower path $P C$, and the upper path $C D$. If we connect $D$ with $Q$ by a suitable lower path (i.e., the dotted oriented lower path $D Q$ in Figure 4.2), we get the 2-component link drawn in Figure 4.3(a). The sequence of Reidemeister moves in Figure 4.3 proves that this link is equivalent to the Hopf link. So the manifold $M^{3}$ is the real projective 3-space.

The Heegaard diagram in Figure 4.1 determines a further balanced geometric presentation for $\pi_{1}\left(M^{3}\right) \cong \mathbb{Z}_{2}$ with three generators $X, Y$, and $Z$ corresponding to meridians 


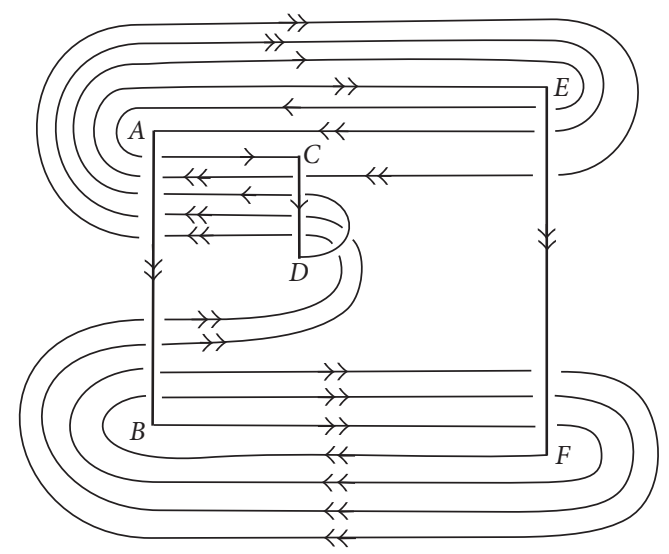

(a)

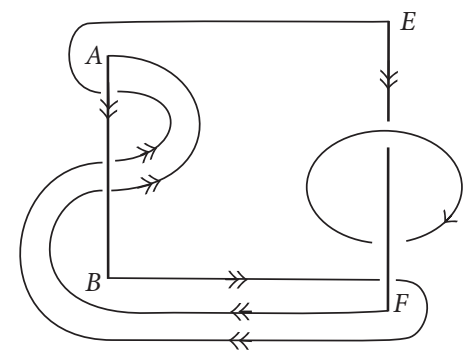

(c)

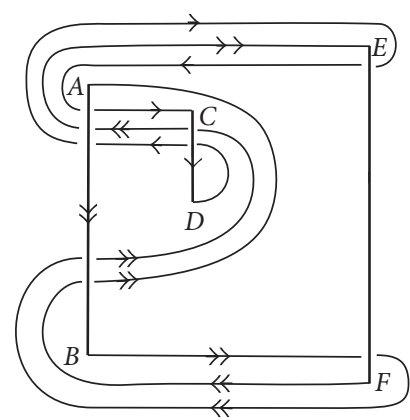

(b)

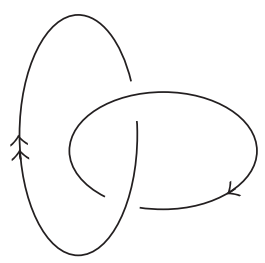

(d)

Figure 4.3. A sequence of Reidemeister moves yielding the Hopf link.

$u_{1}, u_{2}$, and $u_{3}$, respectively, and three relations

$$
\begin{gathered}
Y Z\left(Y Z^{-1} Y X^{-1} Y Z^{-1}\right)^{2}=1, \\
Y X^{-1} Y Z^{-1} Y X^{-1} Y Z^{-1} Y^{2} Z^{-1} Y X^{-1} Y Z^{-1}=1, \\
Y X^{-1} Z Y^{-1} X Y^{-1} Z Y^{-1} Z^{-1} Y Z^{-1} Y^{-1} Z Y^{-1} X Y^{-1} Z X^{-1} Y Z^{-1}=1,
\end{gathered}
$$

which correspond to longitudes.

\section{Irreducible Heegaard diagrams of connected sums}

Repeating $n$ times certain arcs of the Im-Kim diagram, as indicated in Figure 4.1, we get a family of irreducible Heegaard diagrams of genus three for closed orientable 3-manifolds $M(n)$. Such diagrams are 2-symmetric, and represent $M(n)$ as 2-fold branched coverings of the 3-sphere. Determining the branch set as described in the previous sections, one obtains the one-linked union of a trivial knot and a 2-bridge knot $\mathbf{b}(2 n-1,1)$. So the manifold $M(n)$ is homeomorphic to $\mathbb{R} P^{3} \# L(2 n-1,1)$, for any $n \geq 1$ (use also Proposition 5.2 
below). Let $X, Y$, and $Z$ be the generators of the fundamental group of $M(n)$ which are represented by the meridians $u_{1}, u_{2}$, and $u_{3}$, respectively. Walking along the longitudes, coherently with their orientations, yields the relations (listed below) of a balanced presentation for $\pi_{1}(M(n)) \cong \mathbb{Z}_{2} * \mathbb{Z}_{2 n-1}$. More precisely, we have the following proposition.

Proposition 5.1. For every $n \geq 1$, the group $\mathbb{Z}_{2} * \mathbb{Z}_{2 n-1}$ (where $\mathbb{Z}_{1}=\{1\}$ ) admits a balanced presentation with generators $X, Y$, and $Z$, and relations

$$
\begin{gathered}
\left(Y Z^{-1}\right)^{2} Y X^{-1}\left(Y Z^{-1}\right)^{n} Y Z\left(Y Z^{-1}\right)^{n} Y X^{-1}=1, \\
\left(Y X^{-1} Y Z^{-1}\right)^{2} Y X^{-1}\left(Y Z^{-1}\right)^{n} Y\left(Y Z^{-1}\right)^{n}=1, \\
Y X^{-1} Z Y^{-1} X Y^{-1}\left(Z Y^{-1}\right)^{n} Z^{-1} Y Z^{-1} Y^{-1}\left(Z Y^{-1}\right)^{n} X Y^{-1} Z X^{-1} Y Z^{-1}=1 .
\end{gathered}
$$

This presentation is geometric, that is, it corresponds to a Heegaard diagram (or, equivalently, to a spine) of the manifold $\mathbb{R} P^{3} \# L(2 n-1,1)$.

To complete the paper we give the following result which is similar to that of [16, Proposition 5-5], concerning with the manifold $\left(\mathbb{S}^{1} \times \mathbb{S}^{2}\right) \# L(p, q)$. The proof proceeds in the same way but we include it to make the reading clear.

Proposition 5.2. The 2-fold covering of $\mathbb{S}^{3}$ branched over a link is homeomorphic to $\mathbb{R} P^{3} \# L(p, q), p$ odd, if and only if the link is equivalent to a one-linked union of a trivial $k$ not and a 2-bridge knot $\mathbf{b}(p, q)$.

Proof. Let $M^{3}(L)$ denote the 2-fold covering of $\mathbb{S}^{3}$ branched over a link $L$. Then $M^{3}(L)=$ $M^{3}\left(L_{1}\right) \# M^{3}\left(L_{2}\right) \#\left(\mathbb{S}^{1} \times \mathbb{S}^{2}\right)$ if $L$ is a splittable union $L_{1} \cup L_{2}$, and $M^{3}(L)=M^{3}\left(L_{1}\right) \# M^{3}\left(L_{2}\right)$ if $L$ is a connected sum $L_{1} \# L_{2}$. Take $M^{3}\left(L_{1}\right)=\mathbb{R} P^{3}$ and $M^{3}\left(L_{2}\right)=L(p, q)$ in the second case. Sufficiency is an immediate consequence of a theorem of Hodgson [9] (see also [25]), which says that the 2 -fold covering of $\mathbb{S}^{3}$ branched over a link is homeomorphic to a lens space $L(p, q), p \neq 0$, if and only if the link is equivalent to a 2-bridge link $\mathbf{b}(p, q)$. In our case, $L_{1}=\mathbf{b}(2,1)$ is the Hopf link, and $L_{2}=\mathbf{b}(p, q), p$ odd, is the 2-bridge knot of type $(p, q)$. In order to show the necessity, we will apply the $\mathbb{Z}_{2}$-equivariant sphere theorem [12] to the covering translation $\tau$ on $M^{3}(L)=\mathbb{R} P^{3} \# L(p, q)$. Since $M^{3}(L)$ is not irreducible there is a $\tau$-equivariant 2 -sphere $\mathbb{S}^{2}$ in $M^{3}(L)$ which bounds no 3-ball, and the projection of $\mathbb{S}^{2}$ decomposes $L$ into either $L_{1} \cup L_{2}$ or $L_{1} \# L_{2}$. By the uniqueness of prime decompositions [13] we obtain $M^{3}\left(L_{1}\right)=\mathbb{R} P^{3}$ and $M^{3}\left(L_{2}\right)=L(p, q)$. By [9] we can conclude that $L$ is a one-linked union of a trivial knot and a 2-bridge $\operatorname{knot} \mathbf{b}(p, q)$.

\section{Acknowledgments}

The present work was performed under the auspices of the GNSAGA of the CNR (National Research Council) of Italy within the project "Proprietà Geometriche delle Varietà Reali e Complesse,” partially supported by MIUR (Ministero dell'Istruzione, dell'Università e della Ricerca) of Italy, and by a research grant of the University of Modena and Reggio Emilia (Italy). 


\section{References}

[1] J. S. Birman and H. M. Hilden, Heegaard splittings of branched coverings of $\mathbb{S}^{3}$, Transactions of the American Mathematical Society 213 (1975), 315-352.

[2] A. Cavicchioli, Neuwirth manifolds and colourings of graphs, Aequationes Mathematicae 44 (1992), no. 2-3, 168-187.

[3] A. Cavicchioli, D. Repovš, and A. B. Skopenkov, Open problems on graphs arising from geometric topology, Topology and Its Applications 84 (1998), no. 1-3, 207-226.

[4] M. Ferri, Crystallisations of 2-fold branched coverings of $\mathbb{S}^{3}$, Proceedings of the American Mathematical Society 73 (1979), no. 2, 271-276.

[5] M. Ferri, C. Gagliardi, and L. Grasselli, A graph-theoretical representation of PL-manifolds - a survey on crystallizations, Aequationes Mathematicae 31 (1986), no. 2-3, 121-141.

[6] A. T. Fomenko and S. V. Matveev, Algorithmic and Computer Methods for Three-Manifolds, Mathematics and Its Applications, vol. 425, Kluwer Academic, Dordrecht, 1997.

[7] C. Gagliardi, Extending the concept of genus to dimension n, Proceedings of the American Mathematical Society 81 (1981), no. 3, 473-481.

[8] J. Hempel, 3-Manifolds, Princeton University Press, New Jersey, 1976.

[9] C. D. Hodgson, Involutions and isotopies of lens spaces, Master's thesis, University of Melbourne, Melbourne, 1981.

[10] T. Homma, M. Ochiai, and M. Takahashi, An algorithm for recognizing $\mathbb{S}^{3}$ in 3-manifolds with Heegaard splittings of genus two, Osaka Journal of Mathematics 17 (1980), no. 3, 625-648.

[11] Y. H. Im and S. H. Kim, An irreducible Heegaard diagram of the real projective 3-space $\mathbf{P}^{3}$, International Journal of Mathematics and Mathematical Sciences 23 (2000), no. 2, 123-129.

[12] P. K. Kim and J. L. Tollefson, Splitting the PL involutions of nonprime 3-manifolds, The Michigan Mathematical Journal 27 (1980), no. 3, 259-274.

[13] J. Milnor, A unique decomposition theorem for 3-manifolds, American Journal of Mathematics 84 (1962), 1-7.

[14] O. Morikawa, A counterexample to a conjecture of Whitehead, Mathematics Seminar Notes. Kobe University 8 (1980), no. 2, 295-298.

[15] S. Negami, The minimum crossing of 3-bridge links, Osaka Journal of Mathematics 21 (1984), no. 3, 477-487.

[16] S. Negami and K. Okita, The splittability and triviality of 3-bridge links, Transactions of the American Mathematical Society 289 (1985), no. 1, 253-280.

[17] M. Ochiai, A counterexample to a conjecture of Whitehead and Volodin-Kuznetsov-Fomenko, Journal of the Mathematical Society of Japan 31 (1979), no. 4, 687-691.

[18] _ A Heegaard-diagram of the 3-sphere, Osaka Journal of Mathematics 22 (1985), no. 4, 871-873.

[19] M. Pezzana, Sulla struttura topologica delle varietà compatte, Atti del Seminario Matematico e Fisico dell'Università di Modena 23 (1974), no. 1, 269-277.

[20] _ Diagrammi di Heegaard e triangolazione contratta, Bollettino della Unione Matematica Italiana. Supplemento 12 (1975), no. 3, 98-105.

[21] D. Repovš, N. B. Brodskij, and A. B. Skopenkov, A classification of 3-thickenings of 2-polyhedra, Topology and Its Applications 94 (1999), no. 1-3, 307-314.

[22] D. Rolfsen, Knots and Links, Mathematics Lecture Series, no. 7, Publish or Perish, California, 1976.

[23] J. Singer, Three-dimensional manifolds and their Heegaard diagrams, Transactions of the American Mathematical Society 35 (1933), no. 1, 88-111.

[24] M. Takahashi, Two knots with the same 2-fold branched covering space, Yokohama Mathematical Journal 25 (1977), no. 1, 91-99.

[25] J. L. Tollefson, Involutions on $\mathbb{S}^{1} \times \mathbb{S}^{2}$ and other 3-manifolds, Transactions of the American Mathematical Society 183 (1973), 139-152. 
[26] O. Ja. Viro and V. L. Kobel'skiü, The Volodin-Kuznetsov-Fomenko conjecture on Heegaard diagrams is false, Uspekhi Matematicheskikh Nauk 32 (1977), no. 5(197), 175-176 (Russian).

[27] I. A. Volodin, V. E. Kuznetsov, and A. T. Fomenko, The problem of discriminating algorithmically the standard three-dimensional sphere, Russian Mathematical Surveys 29 (1974), no. 5, 71-172.

[28] J. H. C. Whitehead, On certain sets of elements in a free group, Proceedings of the London Mathematical Society 41 (1936), 48-56.

Alberto Cavicchioli: Dipartimento di Matematica, Università di Modena e Reggio Emilia, Via Campi 213/B, 41100 Modena, Italy

E-mail address: cavicchioli.alberto@unimo.it

Fulvia Spaggiari: Dipartimento di Matematica, Università di Modena e Reggio Emilia, Via Campi 213/B, 41100 Modena, Italy

E-mail address: spaggiari.fulvia@unimo.it 


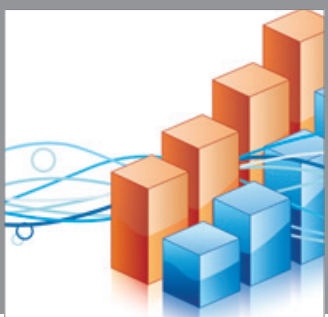

Advances in

Operations Research

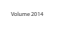

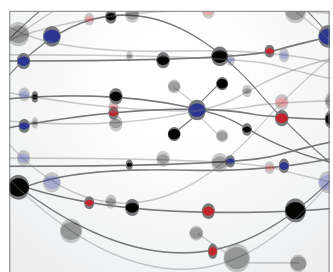

\section{The Scientific} World Journal
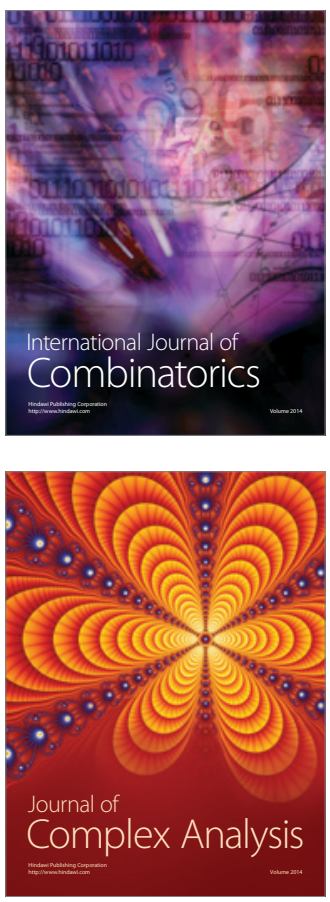

International Journal of

Mathematics and

Mathematical

Sciences
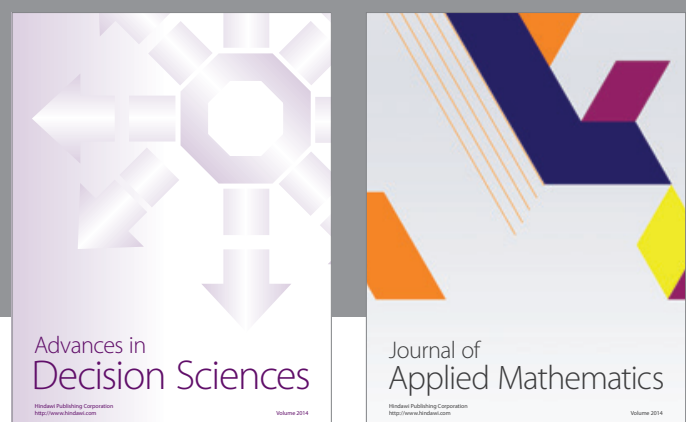

Journal of

Applied Mathematics
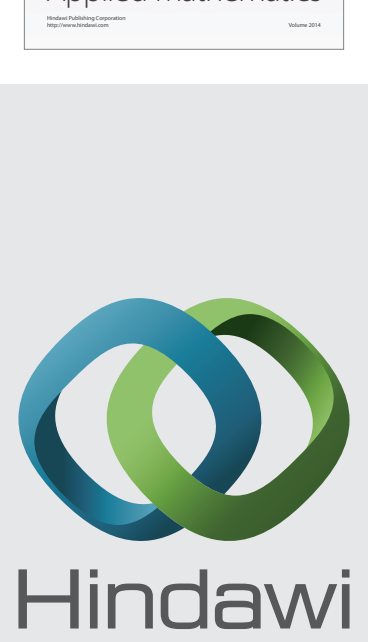

Submit your manuscripts at http://www.hindawi.com
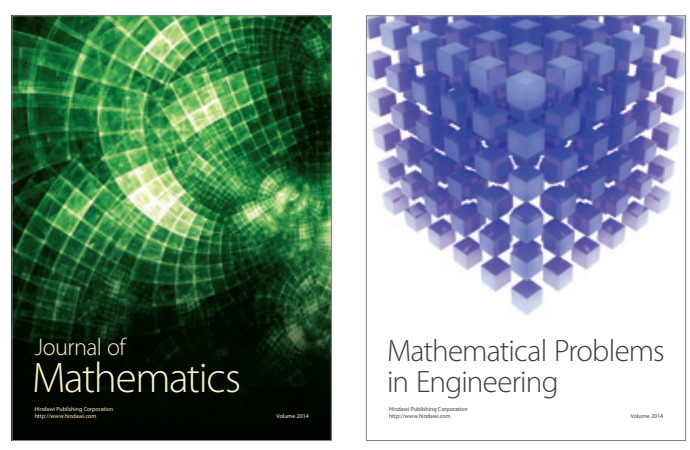

Mathematical Problems in Engineering
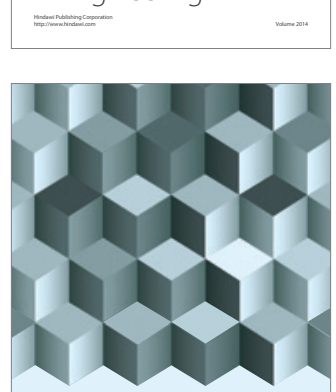

Journal of

Function Spaces
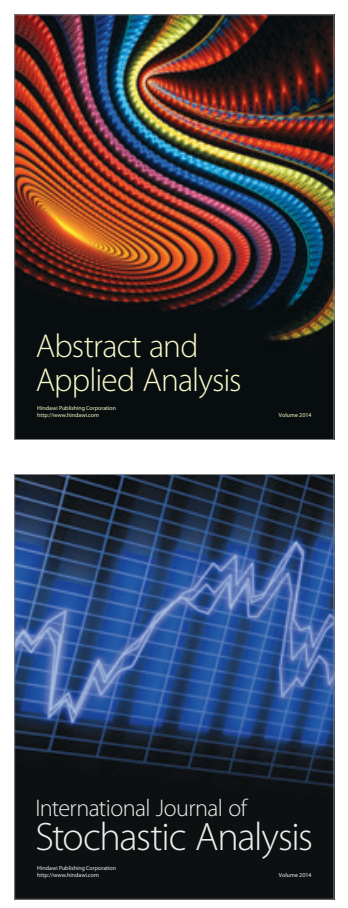

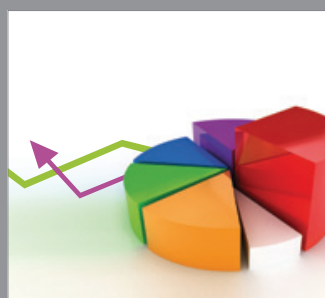

ournal of

Probability and Statistics

Promensencen
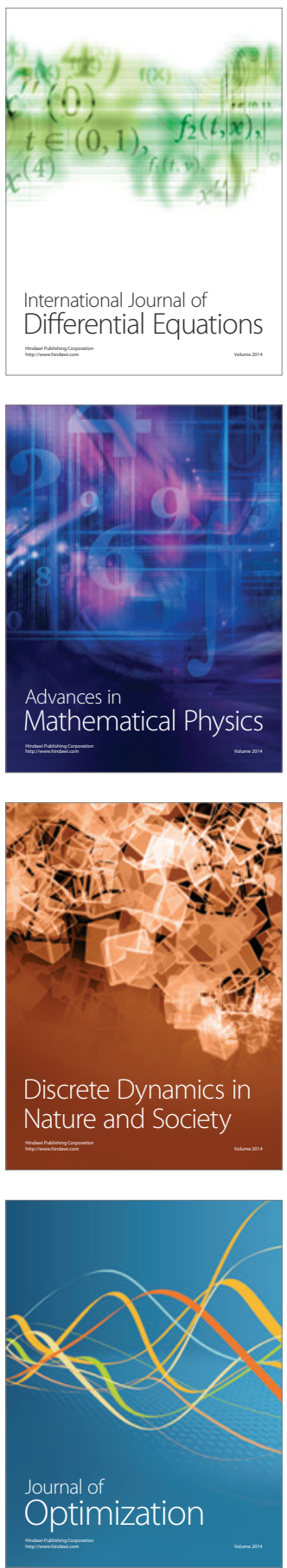